\title{
Enhanced Phase Retrieval Method Based on Random Phase Modulation
}

\author{
Fanxing $\mathrm{Li}^{1,2}$, Wei Yan ${ }^{2, *} \mathbb{C}$, Fupin Peng ${ }^{1,2}$, Simo Wang ${ }^{1,2}$ and Jialin Du ${ }^{1,2}$ \\ 1 The School of Electronic, Electrical and Communication Engineering, University of Chinese Academy of \\ Sciences, Beijing 100049, China; lifanxing15@mails.ucas.edu.cn (F.L.); pengfuping16@mails.ucas.ac.cn (F.P.); \\ wangsimo18@mails.ucas.ac.cn (S.W); dujialin18@mails.ucas.ac.cn (J.D.) \\ 2 Institute of Optics and Electronics, Chinese Academy of Science, Chengdu 610209, China \\ * Correspondence: yanwei@ioe.ac.cn
}

Received: 17 January 2020; Accepted: 3 February 2020; Published: 10 February 2020

check for updates

\begin{abstract}
The phase retrieval method based on random phase modulation can wipe out any ambiguity and stagnation problem in reconstruction. However, the two existing reconstruction algorithms for the random phase modulation method are suffering from problems. The serial algorithm from the spread-spectrum phase retrieval method can realize rapid convergence but has poor noise immunity. Although there is a parallel framework that can suppress noise, the convergence speed is slow. Here, we propose a random phase modulation phase retrieval method based on a serial-parallel cascaded reconstruction framework to simultaneously achieve quality imaging and rapid convergence. The proposed serial-parallel cascaded method uses the phased result from the serial algorithm to serve as the initialization of the subsequent parallel process. Simulations and experiments demonstrate that the superiorities of both serial and parallel algorithms are fetched by the proposed serial-parallel cascaded method. In the end, we analyze the effect of iteration numbers from the serial process on the reconstruction performance to find the optimal allocation scope of iteration numbers.
\end{abstract}

Keywords: random phase modulation; phase retrieval; spatial light modulator

\section{Introduction}

The profile and inner structure of an object lie in the complex field scattered from the object. However, current detectors are only sensitive to amplitude, leaving the phase information unrecorded. The problem that remains to be solved is how to get the phase information of a wavefront, because of the lack of apparatus with a response frequency faster than the wave frequency. Interferometric imaging approaches use a known reference wave to hide the phase information in the interference pattern produced by the reference and target wavefront $[1,2]$. However, the existence of extra reference light increases the complexity and disturbance of the system [3]. Beam-propagation-based approaches are another powerful tool to reconstruct the complex amplitude of sample. The simple experimental set-up makes this technique capable of robustness to external influence and suitability for various wavelengths [4]. The beam-propagation-based methods utilize variants of iterative phase retrieval techniques which originate from the Gerchberg-Saxton (GS) algorithm to recover the complete wavefront [5]. The iterative phase retrieval works via numerical propagation of the light field back and forth between the recording plane and the object plane. The iterative methods which require only a single measurement always suffer from low convergence speed and much sensitivity to the initial value [6-8]. Although successful reconstruction could be obtained with these methods, the difficulties encountered in obtaining a tight support constraint limit the application scope.

To overcome these restrictions, multi-image phase retrieval techniques that use different kinds of diversities in data collection were proposed [9-21]. Methods to generate multiple-diversity 
intensity measurements include, among others, illumination area overlap $[9,10]$, multiple recording distance [11-14], multiple wavelength [15], and illumination beam tilting [16,17]. Except for the multiple wavelength method, the other three types of methods require employing mechanical platforms, which inevitably lead to low acquisition speed and cause potential mechanical error. The method which uses varied wavelength to offer diversities may lead to stagnation and ambiguity in the iterative process due to the lack of intensity variation when the wavelength changes are relatively small. In fact, random phase modulation can provide effective diversity to realize quality imaging results in multi-image phase retrieval methods. Since the "uniqueness" of the random phase can break any symmetry that may exist in the object field, it allows for wiping out any ambiguity and stagnation problem [22,23]. Furthermore, variable modulation can be implemented using a spatial light modulator (SLM); thus, data acquisition speed is improved and mechanical error is avoided. The spread-spectrum phase retrieval (SSPR) method can realize rapid convergence rate by using random phase modulation and a serial reconstructed framework [20,21]. However, the introduction of random phase modulation and a coherent light source brings noise, and the SSPR accumulates noise as the iteration progresses since the serial reconstruction algorithm has no noise suppression operation. A parallel reconstructed framework proposed in Reference [19] can be used in the random phase modulation method to enhance the noise immunity and robustness, due to the existence of an average operation in every iteration. Nevertheless, the parallel reconstructed algorithm suffers from slow convergence speed.

In this study, we propose a beam-propagation-based phase retrieval method based on random phase modulation and a serial-parallel cascaded reconstruction framework, which can suppress the noise generated during the imaging process while realizing rapid convergence. In the proposed method, we use the serial algorithm from SSPR to generate an initial guess of the parallel reconstruction algorithm. Since an acceptable initial guess is obtained from a few iterations of the serial framework, the needed iteration number of the whole reconstruction process is reduced, such that the convergence rate is improved. The noise immunity and stability of the method are enhanced by the subsequent iterations of the parallel algorithm. Accordingly, the serial-parallel concatenation framework can realize a rapid convergence rate and quality imaging results in noisy environments. In this work, the validity of the proposed method is confirmed both theoretically and experimentally. Then, we analyze the impact of different allocation schemes with iteration numbers of serial processes on the reconstruction performance.

\section{Methods}

In the beam-propagation-based random phase modulated methods, the variable random phase modulations are produced by an SLM which is inserted between the object plane and the recording plane, as displayed in Figure 1. The diffraction pattern $I_{m}$, which is modulated by the random phase $\varphi_{m}$, is collected by a charge-coupled device (CCD), where $m=1,2, \ldots, M$, and $M$ is the number of measurement intensities. The distance between the object and SLM plane is denoted as Z1, and that between the SLM and CCD plane is Z2.

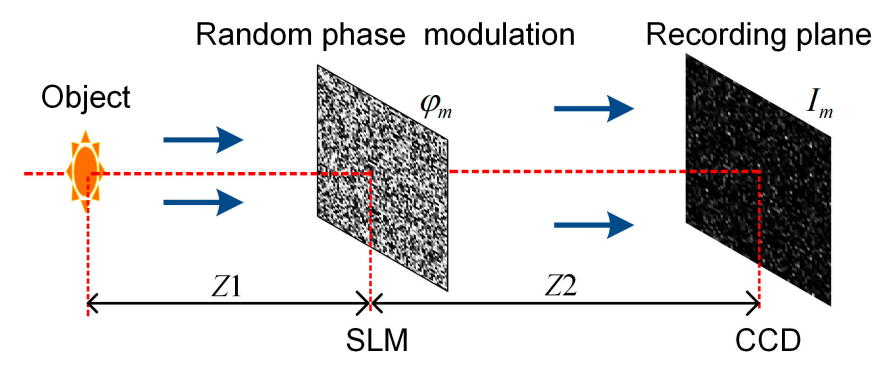

Figure 1. Schematic of the optical set-up. $\varphi_{m}$ is the modulated random phase, and $I_{m}$ is the corresponding recoded intensity. 
The algorithm architectures of the serial and parallel frameworks are outlined in Figure 2a,b, respectively. The iterations of the two algorithms begin with the plane before SLM, denoted by $U_{n}$, which contains the diffraction information of the targeted object, where $n$ is the iteration number. Additionally, $U_{N}$ is the result after $N$ iterations, and the reconstructed object field is calculated by propagation $U_{N}$ back to the object plane, which is realized by the angular spectrum propagation operator $\mathbf{D}_{Z 1}^{-1}$, where the subscript is the diffractive distance.

(a)



(b)

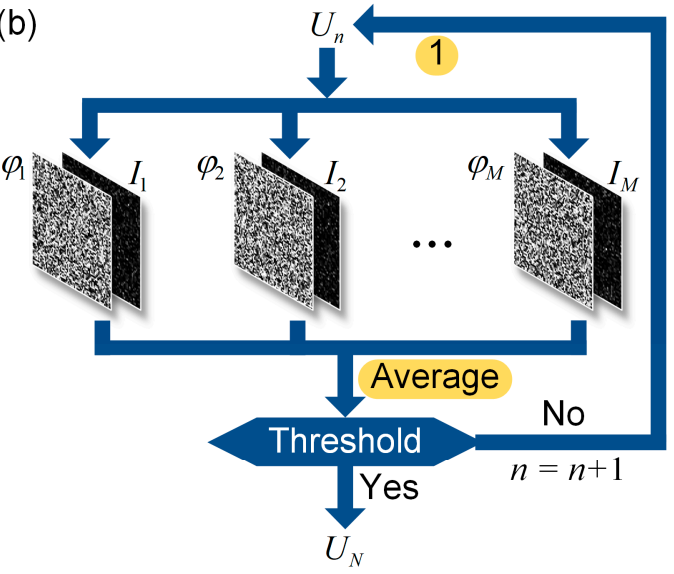

Figure 2. Figure 2. Reconstruction framework of serial and parallel algorithms: (a) the serial framework; (b) the parallel framework. Numbers in the yellow circles indicate the update times of $U_{n}$ in one iteration.

In every iteration of the serial framework, $U_{n}$ is sequentially refined by $M$ pairs of modulated random phases $\varphi_{1}, \varphi_{2}, \ldots, \varphi_{M}$ and the corresponding recoded intensities $I_{1}, I_{2}, \ldots, I_{M}$. As the numbers in yellow circles shown in Figure 2a, the updates of $U_{n}$ from the first pair of data $I_{1}$ and $\varphi_{1}$ to the last pair of $I_{M}$ and $\varphi_{M}$ perform $M$ times in one iteration. Accordingly, the serial framework holds the ability of rapid convergence. Nevertheless, the stability is affected since every update uses only one pair of data. In addition, the noise immunity of the serial method is poor, because there is no anti-noise action in the reconstruction process. More importantly, the introduction of SLM brings about random modulation noise, the use of a coherent light source generates speckle noise, and dark current noise exists in the CCD. As a result, the quality of the reconstructed results decreases in practical application. In the parallel framework, the average of the refined results obtained by all the pairs of the measurements and the corresponding random phases separately is used to update $U_{n}$. Thus, the robustness and noise immunity are improved. The average operation has the ability to work as a low-pass filter to suppress noise. However, the convergence speed of the parallel framework is reduced since the $U_{n}$ is updated only once in every iteration, as the number in the yellow circle shown in Figure $2 b$.

The refinement procedure of $U_{n}$ using the $m$-th pair of random phase $\varphi_{m}$ and the corresponding intensity $I_{m}$ is illustrated in Figure 3. The refinement procedure proceeds with the following steps:

1. $\quad U_{n}$ is modulated by the random phase $\varphi_{m}$, yielding a modulated field $U_{n} \exp \left(j \varphi_{m}\right)$;

2. The modulated field is numerically propagated into the recording plane by the angular spectrum propagation operator $\mathbf{D}_{\mathrm{Z2}}$;

3. The measurement intensity $I_{m}$ is used to replace the magnitude of the calculated distribution of recording plane;

4. After the replacement, the field is propagated back to the SLM plane by $\mathbf{D}_{Z 2}^{-1}$;

5. The random phase modulation in step (1) is removed to generate a refined estimation of $U_{n}$.

The refinement procedure can be applied to both serial and parallel frameworks. 


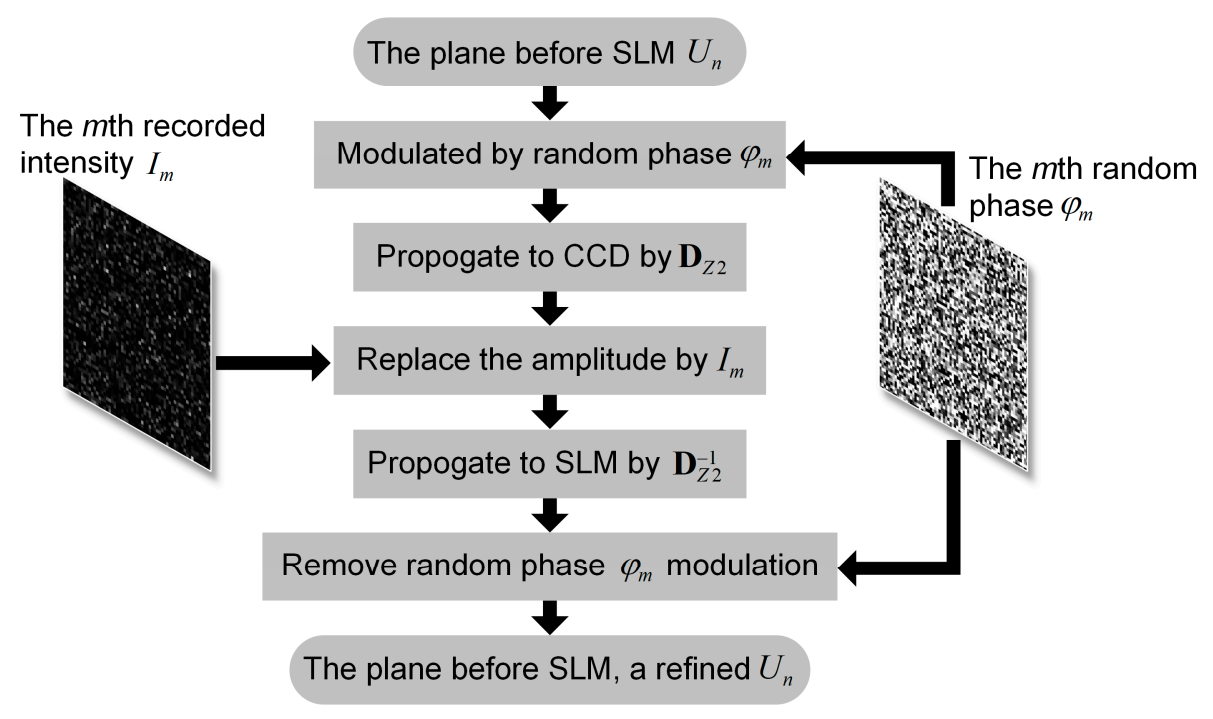

Figure 3. The flow chart of the refinement procedure of $U_{n}$.

The proposed method combines the serial and parallel reconstruction framework to simultaneously guarantee noise immunity and convergence speed, which can be divided into two steps: (a) using the serial reconstruction algorithm to obtain an acceptable phase estimation; (b) using the estimation as an initialization to carry out the iterations of the parallel algorithm. We use a few iterations from the serial algorithm to replace a large number of iterations from the parallel algorithm in the earlier stage of the reconstruction process. Subsequently, the robustness and the ability of noise suppression are enhanced by the parallel framework. Accordingly, the proposed serial-parallel cascaded reconstruction method can achieve both quality reconstruction results and rapid convergence. The iteration number of the proposed method is the sum of serial and parallel processes iterations. We use $n_{1}$ and $n_{2}$ to denote the iteration numbers of the serial and parallel processes, respectively, as $n_{1}+n_{2}=\hat{N}$, and $\hat{N}$ is the eventual iteration number of the serial-parallel cascaded method.

\section{Results}

To evaluate the performance of the two existing algorithms and the proposed serial-parallel cascaded approach, we simulated the reconstruction processes using a two-dimensional complex object. As shown in Figure 4, images "einstein" and "galaxia" were used for the amplitude and phase of the simulated object, respectively, with a resolution of $1024 \times 1024$ pixels. A "distant planet" shown in the sub-image of Figure $4 \mathrm{~b}$ is used as a prominent comparison for the reconstructed results. We use the following parameters: wavelength $=532 \mathrm{~nm}$, pixel size of the recording plane $=5.5 \mu \mathrm{m}$, distance from the object plane to SLM $=100 \mathrm{~mm}$, and distance between SLM and the CCD plane $=150 \mathrm{~mm}$. In the simulation, four pairs of random phases and the corresponding measurement intensities are reconstructed. 

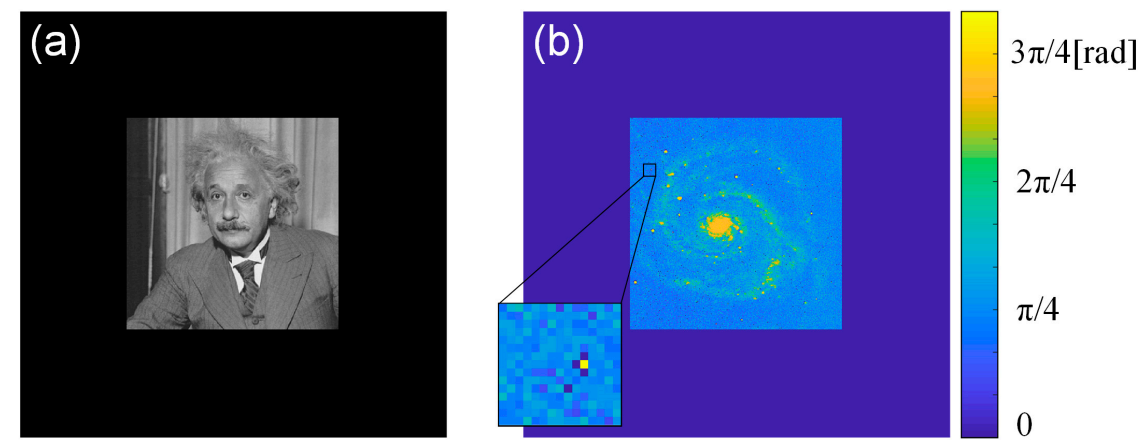

Figure 4. The complex amplitude of the simulated object: (a) the amplitude; (b) the phase.

Firstly, we evaluate the convergence speed of the serial, parallel, and the proposed serial-parallel cascaded methods. In the reconstruction process, the iteration numbers of the three methods were set to 50 , and the iteration number in the cascaded method was allocated as follows: $n_{1}=5$, $n_{2}=45$. Figure 5a plots relationships between iteration numbers and mean square errors (MSE) of the reconstructed amplitudes from these three methods. MSE is defined as

$$
\operatorname{MSE}=\frac{1}{X Y} \sum_{\forall x, y}\left[\left|O_{n}(x, y)\right|-\left|O_{\text {real }}(x, y)\right|\right]^{2},
$$

where $O_{n}$ is the reconstructed object distribution after the $n$-th iteration, $n=1,2, \ldots, N$, and $O_{\text {real }}$ represents the real object. $X$ and $Y$ are the pixel numbers of the image, while $x$ and $y$ are the coordinates of the object plane. As shown in Figure 5a, the convergence speeds of serial and cascaded methods were far faster than that of the parallel method. The superiority of the serial algorithm is evidently manifested, while the first five iterations of the proposed cascaded method were the same as the serial method, thereby reducing MSE to 0.0015 . On the other hand, the parallel method required 58 iterations to achieve the same value of MSE. To clearly illustrate the effect of different convergence states on the reconstruction results, the retrieved objects after 50 iterations are displayed in Figure $5 b-g$. We can see that the serial and cascaded methods achieve excellent results after 50 iterations. The results of the parallel framework were affected by noise since there were not enough iterations, and the contrast of the recovered phase was degraded, as illustrated in the sub-image of Figure $5 f$.


Figure 5. The convergence speed curves and the reconstructed objects. (a) The relationship between iteration number and mean square error (MSE) of the reconstructed amplitude from serial, parallel, and the proposed cascaded reconstruction algorithms, $n_{1}=5, N=50$. (b-d) Reconstructed amplitudes of the three methods after 50 iterations; (e-g) corresponding phases. 
We use polluted measurements to assess the noise immunity of the three methods. The iteration numbers were set to 80 to further the parallel method's convergence, and the iteration number allocation scheme of the cascaded method was $n_{1}=5, n_{2}=75$. The noise curves of the three methods are plotted in Figure 6a, where the mean of the added Gaussian noises was $2 \%$ of the average of the four measured intensities. As the variance of the added noise increased, the MSEs of the reconstructed amplitudes increased and the imaging quality degraded. Obviously, the degradation speed of the serial algorithm was faster than that of parallel and cascaded methods. Compared with the cascaded framework, the anti-noise performance of the parallel method was worse when variances of the added noise were less than $46 \%$, since the parallel method did not meet complete convergence, even after 80 iterations. Thus, the imaging quality of the parallel method suffered from the degradation of two aspects: noise and convergence state. When the noise was small, the convergence state played a vital role. The results of the three methods with noise variance of $30 \%$ and a mean of $2 \%$ are illustrated in Figure $6 \mathrm{~b}-\mathrm{g}$. The amplitude result of the serial method displayed the most serious effect of noise. Furthermore, the contrast of the recovered phase of the serial method was affected severely, as we could hardly find the real position of the planet in the sub-image of Figure 6e.

(a)
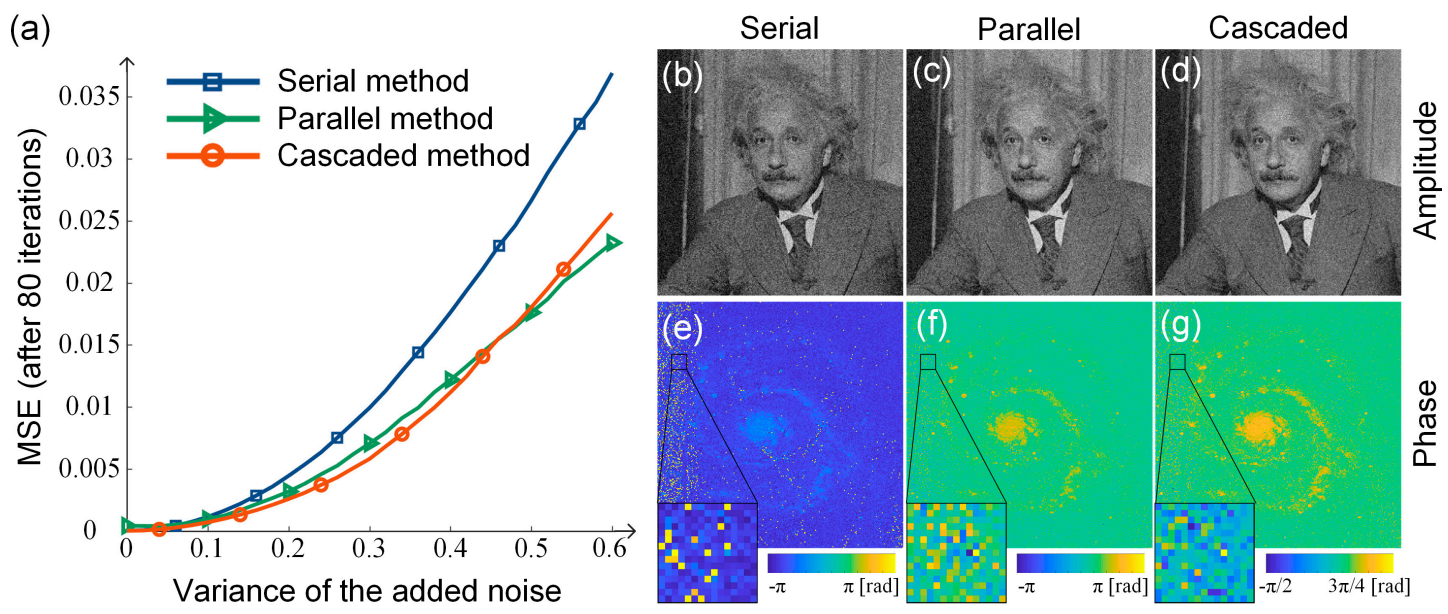

Figure 6. The curves of anti-noise performance and reconstruction results with polluted intensity; $n_{1}=5, N=80$. (a) The relationships between the variances of the added Gaussian noise and MSEs of reconstructed amplitudes from the serial, parallel, and proposed cascaded reconstruction algorithms; (b-d) reconstructed amplitudes with polluted intensities of the three methods after 80 iterations, when the noise variance was 30\% and mean was $2 \%$; (e-g) corresponding recovered phases.

The simulation results demonstrate that the parallel framework had a slow convergence rate and strong noise immunity, while the serial framework had a rapid convergence rate and poor noise immunity. On the other hand, the cascaded approach inherited the merits of both methods, thereby realizing rapid convergence while having a good capability of noise immunity.

\section{Experiment and Analysis}

A lens-free imaging system was used to verify our method, which was composed of four parts: a coherent plane wave $(532 \mathrm{~nm}$ ), a sample, an SLM (pixel size $=12.5 \mu \mathrm{m}$ ), and a CCD camera (pixel size $=3.1 \mu \mathrm{m}$ ). A calibration target etched with a resolution testing board was used for the sample. The distance from the object to the SLM was $100 \mathrm{~mm}$, and that from the SLM to the CCD was $150 \mathrm{~mm}$. Then, four intensity measurements were sequentially recorded by the CCD as the SLM transformed four times. As the image sharpness is a significant evaluation criterion of an image, we used the sharpness 
functions of retrieved images for a global quantification of the convergence rate and reconstruction quality. The sharpness function is defined as follows [24]:

$$
S_{n}=\left[\sum_{\forall x, y}\left|O_{n}(x, y)\right|^{2}\right] /\left[\sum_{\forall x, y}\left|O_{n}(x, y)\right|\right]^{2},
$$

where $S_{n}$ is the sharpness of $O_{n}$, and $O_{n}$ is the reconstruction result after $n$ loops.

The convergence curves after 600 iterations of the three methods are plotted in Figure 7. The serial process of the proposed cascaded method executed 35 iterations, with $n_{1}=35$ and $n_{2}=565$. Although the convergence speed of the serial method was faster than that of the parallel method, the convergent result of the parallel method was much better than that of the serial method. Obviously, only the cascaded method achieved rapid convergence and quality results at the same time. We used the threshold condition ||$S_{n+1}|-| S_{n}|| \leq 1^{-10}$ to extract the convergent iteration numbers to quantify the convergence rates. The iteration numbers and the corresponding sharpness are exhibited in Table 1. Compared with the parallel method, the proposed cascaded method realized the same quality result with fewer iterations.

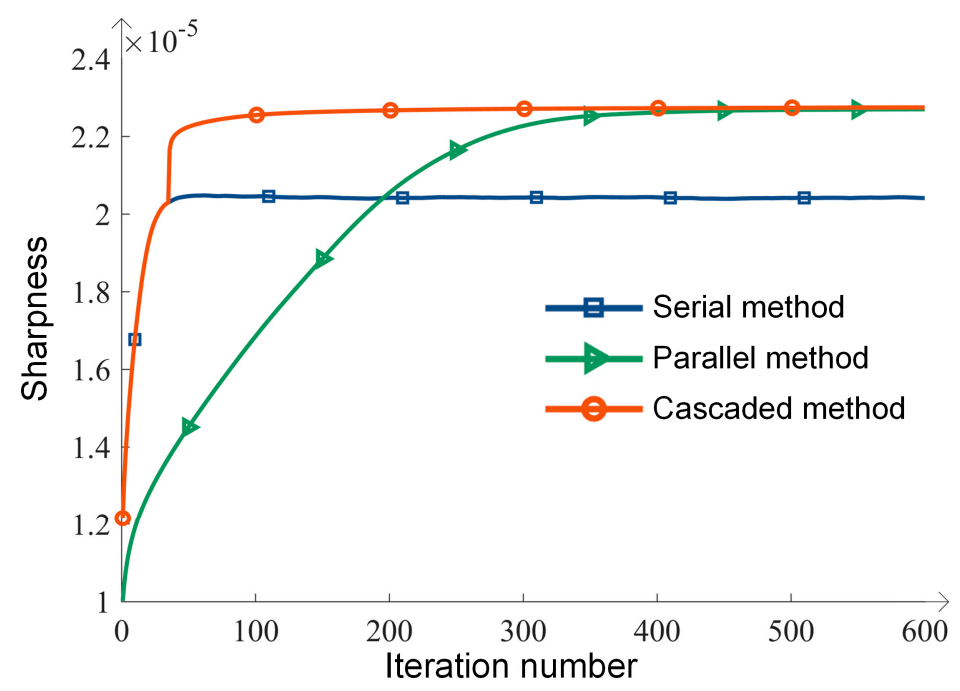

Figure 7. The relationship between iteration number and the sharpness of retrieved amplitudes from the serial, parallel, and the proposed cascaded methods; $n_{1}=35, N=600$.

Table 1. Reconstructed performance comparison of the three methods.

\begin{tabular}{cccc}
\hline Performance & Serial & Parallel & Cascaded \\
\hline Iteration number & 90 & 425 & 156 \\
Sharpness $\left(\times 10^{-5}\right)$ & 2.0475 & 2.2706 & 2.2740 \\
\hline
\end{tabular}

To clearly show the differences in the reconstruction results with different sharpness, the recovery amplitudes and phases of these three methods are shown in Figure 8a-f. In the reconstructed amplitudes exhibited in Figure $8 \mathrm{a}-\mathrm{c}$, the background noise of the serial algorithm was the most severe, compared to the other two methods. Moreover, the data captured from white lines in Figure 8a-c are plotted in Figure 9 to show the detailed distinction of reconstruction quality. Apparently, the contrast of the serial result was reduced by background noise. The parallel and cascaded methods, which held higher sharpness, performed better in terms of reconstruction quality. 

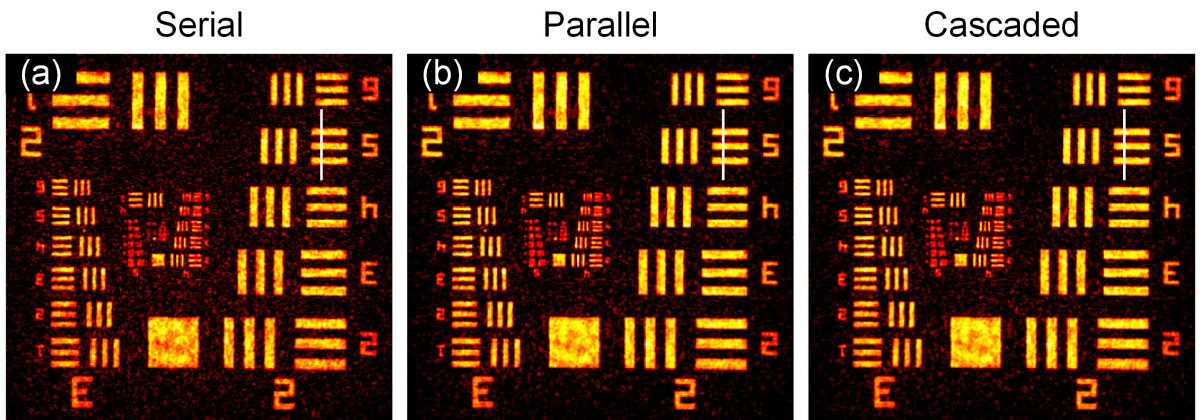

$\frac{\frac{d}{0}}{\frac{\partial}{}}$
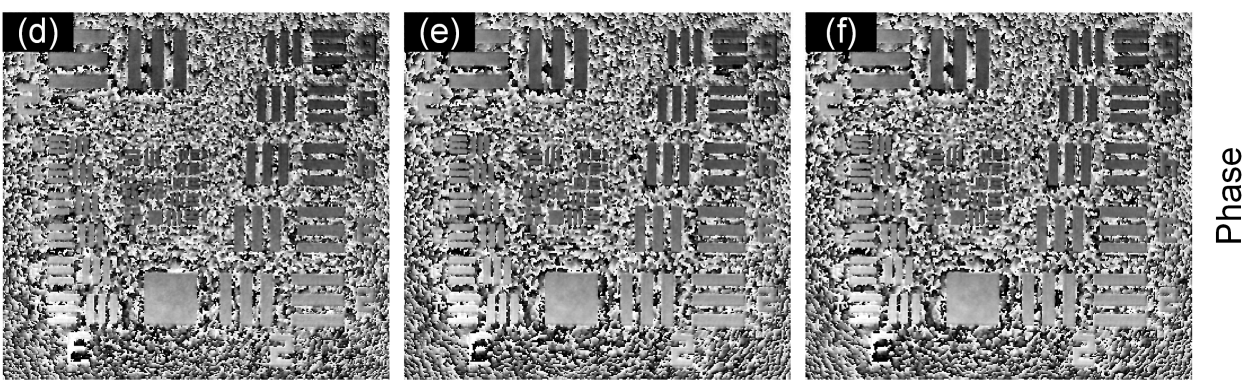

Figure 8. The experimental results. (a-c) Recovered amplitudes of the three methods; $(\mathbf{d}-\mathbf{f})$ reconstructed phases of the three methods.



Figure 9. Cross-sections of the white lines marked in Figure 8a-c.

The experiments show the availabilities of the proposed cascaded method, which extracted the superiorities of both serial and parallel methods, thereby achieving quality imaging with fewer iterations. Nevertheless, there is still an important question to be addressed in the proposed serial-parallel cascaded reconstruction framework. How does the distribution scheme of iteration numbers affect the reconstruction performances? In this work, we mainly analyzed the effect of different iteration numbers of the serial process $n_{1}$ on the iteration number $\hat{N}$ required for convergence and the corresponding reconstruction quality. Furthermore, the threshold condition ||$S_{n+1}|-| S_{n}|| \leq 1^{-10}$ was used to determine the iteration convergence.

The relationship between $n_{1}$ and $\hat{N}$, the number of iterations required for convergence, is plotted with a blue solid curve in Figure 10. With the increase in serial process iteration numbers, the number of iterations required eventually showed a downward trend and increased after encountering a minimum. When the minimum of $\hat{N}$ was achieved, $n_{1}=50$. In the convergence curve of the serial method, the point that the iteration number was equal to 50 can be considered a turning point, since the convergence speed was nearly reduced to 0 after the turning point. The convergence curve of the 
serial method and the corresponding derivative curve are plotted in Figure 11 with a blue solid line and dotted orange line, respectively, while the positions of the turning points are marked with black dots. In the proposed serial-parallel cascaded method, when the iteration number of serial process $n_{1}$ was set to the position of the turning point from the serial convergence curve, the iteration converged at the fastest speed. When the value of $n_{1}$ was smaller than the value of the iteration number of the turning point, more parallel iterations were required later, since the iteration speed of the serial process was much faster than that of the parallel process. When $n_{1}$ was greater than the iteration number of the turning point, any iteration numbers in excess constituted a waste, since the reconstruction quality hardly continued improving.



Figure 10. The effect of the serial process iteration number on reconstruction performance of the proposed cascaded method.

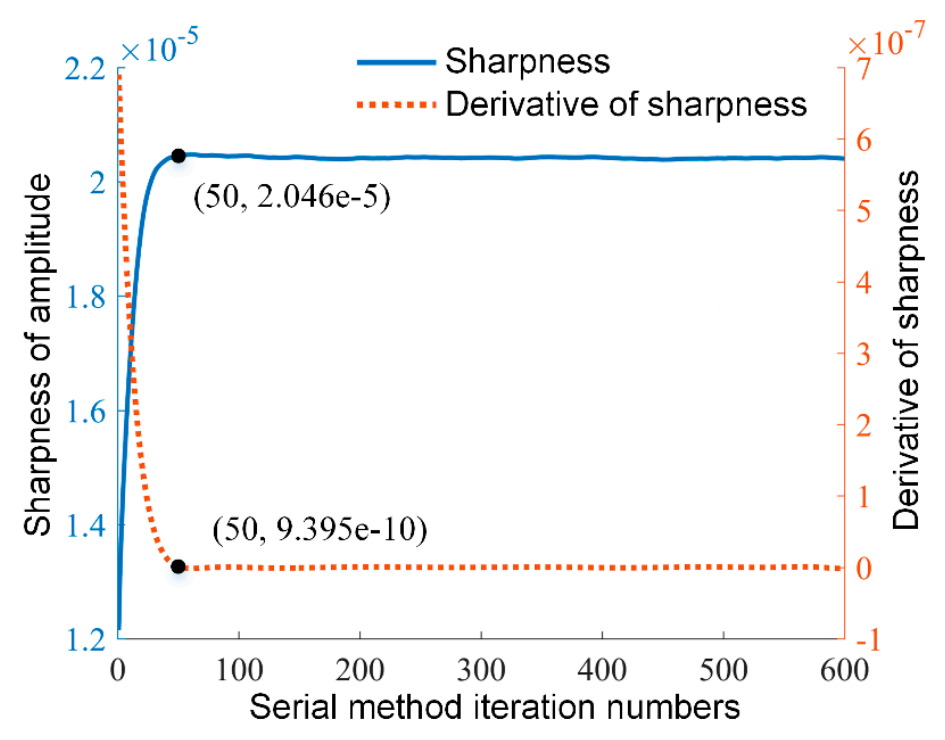

Figure 11. The convergence curve and the corresponding derivative curve of the serial method.

Compared with the convergence speed, the effect of $n_{1}$ on the reconstruction quality of the proposed serial-parallel cascaded method was relatively smaller. The dotted orange line in Figure 10 shows the relationship between serial iteration number $n_{1}$ and the sharpness of the recovered amplitude. Although experimental errors made the relationship curve oscillatory and noisy, we could still find that a continuous increase of $n_{1}$ caused the reconstruction quality to decrease in the convergent state. In particular, when $n_{1}$ was greater than the iteration number of the turning point of the serial 
convergence curve, the degradation was even more serious. Therefore, we recommend that a value before the turning point of the serial method convergence curve should be used to set the iteration number of the serial process $n_{1}$. It is worth noting that $n_{1}$ cannot be too small since an initial result too far away from convergence would severely increase the number of iterations required for the subsequent parallel process.

\section{Conclusions}

In summary, two frameworks were used to reconstruct the beam-propagation-based random phase modulation method: the serial algorithm from SSPR and the parallel algorithm. Although the traditional serial algorithm can converge with a small number of iterations, the anti-noise ability is poor; thus, the reconstruction quality is seriously affected in practical application. On the other hand, the parallel framework has the ability to suppress noise, but the convergence speed is decreased. In this work, we proposed a serial-parallel cascaded framework to achieve quality imaging and rapid convergence of the beam-propagation-based random phase modulated method. The proposed cascaded framework uses an iterative result from the serial algorithm as initialization of the parallel algorithm to simultaneously ensure noise immunity and convergence speed. The simulation and experiment demonstrated the performance of the cascaded method, which inherited the merits of the serial and parallel methods. Furthermore, we analyzed the impact of the initial result from the serial process with different iteration numbers on the reconstruction performance to find the optimum number of iterations of the serial process. The compactness, quick convergence, robustness, and anti-noise properties grant the proposed serial-parallel cascaded random phase modulation method with good potential as an effective tool for wavefront detection and lensless imaging techniques.

Author Contributions: F.L. developed the algorithm; F.L. and F.P. conceptualized and designed the experiments; S.W. and J.D. performed the experiments; F.L., W.Y., and F.P. analyzed the data and wrote the paper; W.Y. applied for the funding and managed the project. All authors read and agreed to the published version of the manuscript.

Funding: This research was funded by the National Natural Science Foundation of China (61705232) and the Instrument Developing Project of the Chinese Academy of Sciences (YA17K014).

Conflicts of Interest: The authors declare no conflicts of interest.

\section{References}

1. Tahara, T.; Quan, X.; Otani, R.; Takaki, Y.; Matoba, O. Digital holography and its multidimensional imaging applications: A review. Microscopy 2018, 67, 55-67. [CrossRef]

2. Marquet, P.; Rappaz, B.; Magistretti, P.J.; Cuche, E.; Emery, Y.; Colomb, T.; Depeursinge, C. Digital holographic microscopy: A noninvasive contrast imaging technique allowing quantitative visualization of living cells with subwavelength axial accuracy. Opt. Lett. 2005, 30, 468-470. [CrossRef]

3. Claus, D.; Hennenlotter, J.; Liting, Q.; Pedrini, G.; Stenzl, A.; Osten, W. Variable Wavefront Curvature Phase Retrieval Compared to Off-Axis Holography and Its Useful Application to Support Intraoperative Tissue Discrimination. Appl. Sci. 2018, 8. [CrossRef]

4. Anand, A.; Javidi, B. Three-dimensional microscopy with single-beam wavefront sensing and reconstruction from speckle fields. Opt. Lett. 2010, 35, 766-768. [CrossRef]

5. Gerchberg, R.W. A practical algorithm for the determination of phase from image and diffraction plane pictures. Optik 1972, 35, 237-250.

6. Fienup, J.R. Phase retrieval algorithms: A comparison. Appl. Opt. 1982, 21, 2758-2769. [CrossRef]

7. Elser, V. Phase retrieval by iterated projections. J. Opt. Soc. Am. A 2003, 20, 40-55. [CrossRef]

8. Fienup, J.R. Phase retrieval algorithms: A personal tour [Invited]. Appl. Opt. 2013, 52, 45-56. [CrossRef]

9. Rodenburg, J.M.; Faulkner, H.M.L. A phase retrieval algorithm for shifting illumination. Appl. Phys. Lett. 2004, 85, 4795-4797. [CrossRef]

10. Faulkner, H.M.; Rodenburg, J.M. Movable aperture lensless transmission microscopy: A novel phase retrieval algorithm. Phys. Rev. Lett. 2004, 93, 023903. [CrossRef] 
11. Pedrini, G.; Osten, W.; Zhang, Y. Wave-front reconstruction from a sequence of interferograms recorded at different planes. Opt. Lett. 2005, 30, 833-835. [CrossRef]

12. Guo, C.; Shen, C.; Tan, J.; Bao, X.; Liu, S.; Liu, Z. A robust multi-image phase retrieval. Opt. Lasers Eng. 2018, 101, 16-22. [CrossRef]

13. Guo, C.; Zhao, Y.; Tan, J.; Liu, S.; Liu, Z. Multi-distance phase retrieval with a weighted shrink-wrap constraint. Opt. Lasers Eng. 2019, 113, 1-5. [CrossRef]

14. Mosso, F.; Peters, E.; Pérez, D.G. Complex wavefront reconstruction from multiple-image planes produced by a focus tunable lens. Opt. Lett. 2015, 40, 4623-4626. [CrossRef]

15. Bao, P.; Zhang, F.; Pedrini, G.; Osten, W. Phase retrieval using multiple illumination wavelengths. Opt. Lett. 2008, 33, 309-311. [CrossRef]

16. Luo, W.; Greenbaum, A.; Zhang, Y.; Ozcan, A. Synthetic aperture-based on-chip microscopy. Light Sci. Appl. 2015, 4, e261. [CrossRef]

17. Hussain, A.; Li, Y.; Liu, D.; Kuang, C.; Liu, X. On-chip Microscopy Using Random Phase Mask Scheme. Sci. Rep. 2017, 7, 14768. [CrossRef]

18. Rodrigo, J.A.; Duadi, H.; Alieva, T.; Zalevsky, Z. Multi-stage phase retrieval algorithm based upon the gyrator transform. Opt. Express 2010, 18, 1510-1520. [CrossRef]

19. Liu, Z.; Guo, C.; Tan, J.; Wu, Q.; Pan, L.; Liu, S. Iterative phase-amplitude retrieval with multiple intensity images at output plane of gyrator transforms. J. Opt. 2015, 17, 025701. [CrossRef]

20. Zhang, F.; Pedrini, G.; Osten, W. Phase retrieval of arbitrary complex-valued fields through aperture-plane modulation. Phys. Rev. A 2007, 75, 043805. [CrossRef]

21. Kohler, C.; Zhang, F.; Osten, W. Characterization of a spatial light modulator and its application in phase retrieval. Appl. Opt. 2009, 48, 4003-4008. [CrossRef]

22. Jesacher, A.; Harm, W.; Bernet, S.; Ritsch-Marte, M. Quantitative single-shot imaging of complex objects using phase retrieval with a designed periphery. Opt. Express 2012, 20,5470-5480. [CrossRef]

23. Fienup, J.R. Reconstruction of a complex-valued object from the modulus of its Fourier transform using a support constraint. J. Opt. Soc. Am. A 1987, 4, 118-123. [CrossRef]

24. Muller, R.A.; Buffington, A. Real-time correction of atmospherically degraded telescope images through image sharpening. J. Opt. Soc. Am. 1974, 64, 1200-1210. [CrossRef] 